\title{
Arbitrary-detuning asynchronous optical sampling pump-probe spectroscopy of bacterial reaction centers
}

\author{
Laura Antonucci, ${ }^{1,2}$ Adeline Bonvalet, ${ }^{1,2}$ Xavier Solinas, ${ }^{1,2}$ Michael R. Jones, ${ }^{3}$ \\ Marten H. Vos, ${ }^{1,2}$ and Manuel Joffre ${ }^{1,2, *}$ \\ ${ }^{1}$ Laboratoire d'Optique et Biosciences, Ecole Polytechnique, Centre National de la Recherche Scientifique, 91128 Palaiseau, France \\ ${ }^{2}$ Institut National de la Santé et de la Recherche Médicale, U696, 91128 Palaiseau, France \\ ${ }^{3}$ School of Biochemistry, Medical Sciences Building, University of Bristol, University Walk, Bristol BS8 1TD, UK \\ ${ }^{*}$ Corresponding author: manuel.joffre@polytechnique.edu
}

Received June 14, 2013; accepted July 19, 2013;

posted August 1, 2013 (Doc. ID 192279); published August 23, 2013

\begin{abstract}
A recently reported variant of asynchronous optical sampling compatible with arbitrary unstabilized laser repetition rates is applied to pump-probe spectroscopy. This makes possible the use of a $5.1 \mathrm{MHz}$ chirped pulse oscillator as the pump laser, thus extending the available time window to almost $200 \mathrm{~ns}$ with a time resolution as good as about $320 \mathrm{fs}$. The method is illustrated with the measurement in a single experiment of the complete charge transfer dynamics of the reaction center from Rhodobacter sphaeroides. @ 2013 Optical Society of America

OCIS codes: (320.7120) Ultrafast phenomena; (320.7160) Ultrafast technology.

http://dx.doi.org/10.1364/OL.38.003322
\end{abstract}

Asynchronous optical sampling (ASOPS) is a widespread technique for rapid time delay scanning, which has found numerous applications, e.g., in time-resolved pump-probe or Fourier-transform spectroscopy. This stroboscopic approach usually relies on two femtosecond oscillators of nearly identical [1-7]—or nearly multiple $[2,8]$ - repetition rates. As compared to more conventional scanning methods based on a mechanical translation stage, the advantages of ASOPS include a faster scanning speed, the removal of pointing instabilities and defocusing for large time delays, the reduction of mechanical noise, and the possibility of achieving longer time measurement windows [1-]].

We have recently reported a new variant of ASOPS, coined arbitrary-detuning ASOPS (AD-ASOPS), which can be more easily applied to any pair of free-running femtosecond oscillators thanks to the lack of any specific requirement on the laser respective repetition rates [9]. In this Letter, we take advantage of this new feature to achieve asynchronous sampling combining for the first time, to the best of our knowledge, a chirped pulse oscillator (CPO) [10], of repetition rate $5.1 \mathrm{MHz}$, with a more conventional oscillator, of repetition rate $74.7 \mathrm{MHz}$. Using the CPO as the pump laser, we are therefore able to extend the available time window to almost $200 \mathrm{~ns}$, while keeping most of the advantages of asynchronous sampling. New systems with longer recombination times-quite common in particular in biological samples - can thus be investigated, such as the reaction center (RC) from Rhodobacter sphaeroides, whose forward electron transfer and charge recombination are studied here by AD-ASOPS time-resolved spectroscopy.

In contrast with conventional ASOPS, the large difference between the two laser repetition rates encountered in AD-ASOPS results in a rapid variation of the pumpprobe time delay. Yet, this variation remains almost perfectly linear (modulo the pump laser period), so that the exact time delay can be interpolated a posteriori. For each digitized probe pulse data point, the corresponding pump-probe delay is computed from the knowledge of the number of probe pulses that elapsed since the last pump-probe coincidence event, associated with the number of pump and probe pulses between the previous and next coincidence events. The data are then averaged in the corresponding time bin so that a complete timeresolved dynamics can be obtained after appropriate data averaging. In a previous AD-ASOPS experiment, we have demonstrated a time resolution of about $400 \mathrm{fs}$, limited by the uncertainty in coincidence detection and by the deviation from the assumed linear evolution resulting from laser timing jitter [9].

Figure 1 shows the experimental setup. The pump laser is a $\overline{\mathrm{CPO}}$ (Femtosource XL 500, Femtolasers), delivering $530 \mathrm{~nJ}, 50 \mathrm{fs}$ pulses at a repetition rate of $5.1 \mathrm{MHz}$, while the probe laser is a chirped-mirror Ti:sapphire oscillator (Synergy PRO, Femtolasers), delivering $4 \mathrm{~nJ}, 15 \mathrm{fs}$ pulses at a repetition rate of $74.7 \mathrm{MHz}$ [11]. Since the two

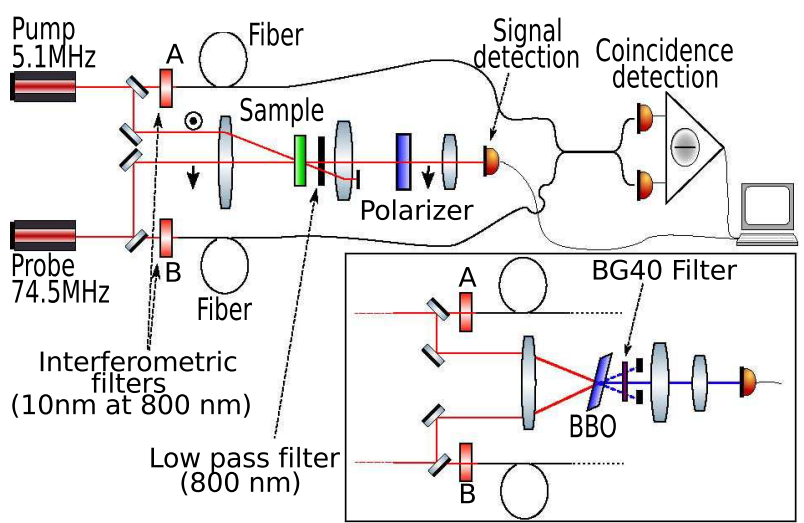

Fig. 1. Experimental setup for AD-ASOPS pump-probe spectroscopy, including the free-space pump-probe experiment and the fiber-based coincidence detection setup. The inset shows the setup used for characterizing the time resolution of the experiment, where a nonlinear BBO crystal is used in place of the sample. 
lasers, both centered at $800 \mathrm{~nm}$, have largely overlapping spectra, coincidence detection can be easily achieved using a fiber-based setup already described elsewhere [9]. The latter measurement relies on the linear interference between the two lasers and is thus highly sensitive, so that only a small fraction of the incident energy is required and is easily provided using the leaked beam transmitted through high-reflection dielectric mirrors. Two interference filters placed before the optical fiber couplers allow enhancement of the spectral overlap, resulting in a coherence time of 210 fs full width at half-maximum (FWHM), assuming a Gaussian profile as deduced from the measured spectra. The coincidence detection threshold, as described in [9], was fixed at $25 \%$ of the maximum interference signal, which results in a theoretical resolution limit of $295 \mathrm{fs}$. The cross-polarized pump and probe pulses are then focused on the sample, and the transmitted probe pulses are individually measured using a photodiode placed after an analyzer rejecting the scattered pump radiation. For each laser shot, the transmitted probe energy and the coincidence signal are acquired simultaneously using a $210 \mathrm{MS} / \mathrm{s}$, 14-bit analog-to-digital acquisition card (X5-210M, Innovative), clocked at the probe-laser frequency.

Note that using a pump laser repetition rate as low as $5.1 \mathrm{MHz}$ results in a significant decrease of the coincidence rate as compared to our previous work [9], increasing the time interval between coincidence events and thus increasing the detrimental effect of timing jitter on time resolution. In order to investigate this effect, we first characterized the time resolution by replacing the sample with a type-I BBO crystal, as shown in the inset of Fig. 1, allowing measurement of the cross-correlation signal and hence of the time resolution of the AD-ASOPS pump-probe setup.

Figure 2 shows the measured autocorrelation curves corresponding to data associated with coincidence pairs separated by less than $1 \mathrm{~ms}$ (red diamonds) or less than $0.5 \mathrm{~ms}$ (blue circles). Indeed, although the average time between coincidence pairs is roughly equal to $5 \mathrm{~ms}$, the random nature of coincidence events resulting from the

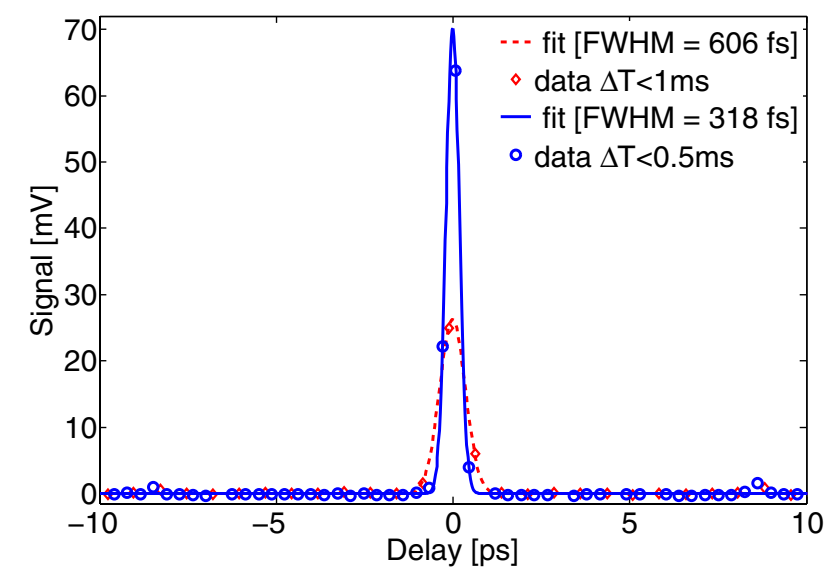

Fig. 2. Cross-correlation signal obtained from sum-frequency mixing in a type-I BBO crystal. The blue circles (resp. red diamonds) are the data measured by selecting coincidence events closer than $0.5 \mathrm{~ms}$ (resp. $1 \mathrm{~ms}$ ). The solid lines correspond to Gaussian fits, indicating a FWHM time resolution of $318 \mathrm{fs}$ (resp. $606 \mathrm{fs})$. mechanical behavior of the two free-running oscillator cavities, associated to a roughly exponentially decaying distribution [9], allows selection of more favorable coincidence pairs. Selecting only coincidence pairs closer than $0.5 \mathrm{~ms}$ (blue dots) results in a time resolution of about $320 \mathrm{fs}$, as deduced from the FWHM of the Gaussian fit. This value is very close to the theoretical limit of 295 fs due to the uncertainty in coincidence detection, indicating that the drift in cavity length during a $0.5 \mathrm{~ms}$ time interval can be safely neglected. In contrast, the data associated with a time interval of $1 \mathrm{~ms}$ between coincidence pairs results in a time resolution greater than $600 \mathrm{fs}$, evidencing the detrimental effect of timing jitter. It is noteworthy that, due to the more restrictive conditions for the first measurement, the acquisition time is about three times longer for the first experiment than for the second one, so that one must compromise between time resolution and acquisition time (or signal-to-noise ratio).

We applied the AD-ASOPS pump-probe setup shown in Fig. 1 to investigate the dynamics of the $P^{+} H_{L}^{-}$charge separated state intermediate in bacterial photosynthesis, by monitoring the formation and decay of the electrochromic band shift of the B band, at $780 \mathrm{~nm}$, close to its induced absorption maximum [12]. The experiment was performed in isolated carotenoid-containing wildtype $R$. sphaeroides RCs, in which the quinone acceptor $Q_{A}$ was prereduced. Under these conditions, the build-up of any long-lived (>200 ns) triplet states of the bacteriochlorophyll dimer electron donor $\mathrm{P}$ is avoided by efficient quenching by the carotenoid [13]. The $1 \mathrm{~mm}$ optical path length sample was placed in a sample holder that was thermostatted at $10^{\circ} \mathrm{C}$ and continuously rotated at $\sim 3 \mathrm{~Hz}$ (diameter $=8 \mathrm{~mm}$ ) in order to minimize sample degradation. The RCs were purified as described elsewhere [14], and were prepared to OD at $800 \mathrm{~nm} \sim 0.5$ in $20 \mathrm{mM}$ Tris, $\mathrm{pH} 8.0,0.1 \% \mathrm{LDAO}$, using $10 \mathrm{mM}$ dithiothreitol as a reductant. The energy of the $50 \mathrm{fs}, 800 \mathrm{~nm}$, s-polarized pump pulses was adjusted to $2 \mathrm{~nJ}$, while the energy of the $15 \mathrm{fs}, 800 \mathrm{~nm}$, p-polarized probe pulses was adjusted to $50 \mathrm{pJ}$. The two beams were focused using an optical lens of focal length $100 \mathrm{~mm}$, and the transmitted probe beam was spectrally filtered using a low-pass filter rejecting wavelengths longer than $800 \mathrm{~nm}$ in order to avoid averaging out of the signal around the isosbestic point. The resulting probe spectrum was centered at $780 \mathrm{~nm}$ with a spectral width of $10 \mathrm{~nm}$ FWHM.

Figure 3 shows the differential absorbance signal obtained using the experimental approach outlined above, using a total acquisition time of only $4 \mathrm{~min}$. The data recorded in the time interval [ $-20 \mathrm{ps}, 40 \mathrm{ps}]$ were subject to a selection keeping only coincidence pairs separated by less than $1 \mathrm{~ms}$ (corresponding as discussed above to a time resolution of $600 \mathrm{fs}$ ), hence the greater amount of noise due to the smaller number of samples. The data are plotted using a linear-logarithmic scale extending up to $196 \mathrm{~ns}$, exhibiting a large variety of time scales in this system. The solid line is a three-exponential fit that yields time constants equal to $2.0 \mathrm{~ns}$ (53\%), $8 \mathrm{~ns}(42 \%)$, and $43 \mathrm{~ns}(5 \%)$. Our results are in good agreement with previously reported results obtained using lowrepetition-rate continuous-probe experiments [타, $\underline{16}]$, where the multiphasic kinetics were interpreted in terms of a gradual stabilization of the $P^{+} H_{L}^{-}$state. Our high time 


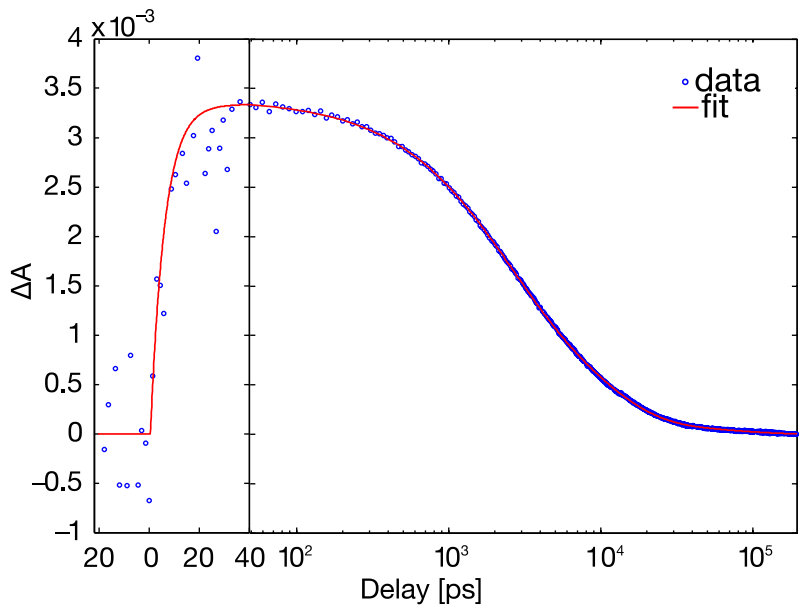

Fig. 3. Time-resolved differential absorbance signal measured in RC. The horizontal axis is linear in the time interval $[-20 \mathrm{ps}$, $40 \mathrm{ps}$ ] and logarithmic in the time interval [40 ps, $196 \mathrm{~ns}$ ]. Blue dots correspond to the data averaged in time bins of width $700 \mathrm{fs}$ in the linear part of the plot and of increasing size in the logarithmic part of the plot, hence the improvement in signal-to-noise ratio with increasing time delay. The solid line shows a fit, with an exponential rising time of $3.7 \mathrm{ps}$ and a three-exponential decay with $2.0 \mathrm{~ns}$ (53\%), 8 ns (42\%), and 43 ns (5\%) time constants.

resolution and excellent signal-to-noise ratio furthermore allow us to more precisely determine the rate and relative amplitude of the fastest component, which presumably reflects recombination from the unrelaxed state. Additionally, our experiment reveals a rise time with a time constant of $3.7 \mathrm{ps}$, also in agreement with previous measurements obtained using conventional ultrafast pumpprobe spectroscopy with a mechanical delay line $[12,17]$.

To summarize, we have applied AD-ASOPS to pumpprobe spectroscopy in a biological system using a $74.7 \mathrm{MHz}$ oscillator combined with a $5.1 \mathrm{MHz}$ CPO. The relatively low repetition rate of the CPO made possible the application of ASOPS to a broad time window extending up to $200 \mathrm{~ns}$, while keeping a best time resolution of about $300 \mathrm{fs}$. We were thus able to measure in a single experiment the complete charge separation and recombination dynamics of carotenoid-containing $R$. sphaeroides RCs. Our results are in good agreement with previous experiments reported earlier but using different experimental approaches suited either to long $[\underline{15}, 16]$ or short [12,17] time scales. Furthermore, our results exhibit an improved signal-to-noise ratio despite an acquisition time of only a few minutes. Finally, we stress that the large energy per pulse available in CPO lasers makes the generation of new spectral components straightfoward, thus opening new perspectives for $\mathrm{AD}$-ASOPS pump-probe spectroscopy in a great variety of spectral domains.

We thank Sergey Laptenok, Michel Negrerie, and Emmanuel Péronne for fruitful discussions and helpful experimental advice. This work was supported by Agence Nationale de la Recherche (ANR-2011-BS04-027).

\section{References and Notes}

1. G. Sucha, M. E. Fermann, D. J. Harter, and M. Hofer, IEEE J. Sel. Top. Quantum Electron. 2, 605 (1996).

2. M. A. Duguay and J. W. Hansen, Appl. Phys. Lett. 13, 178 (1968).

3. E. Lill, S. Schneider, and F. Dorr, Appl. Phys. 14, 399 (1977).

4. P. A. Elzinga, R. J. Kneisler, F. E. Lytle, Y. Jiang, G. B. King, and N. M. Laurendeau, Appl. Opt. 26, 4303 (1987).

5. J. D. Kafka, J. W. Pieterse, and M. L. Watts, Opt. Lett. 17, 1286 (1992).

6. F. Keilmann, C. Gohle, and R. Holzwarth, Opt. Lett. 29, 1542 (2004).

7. A. Bartels, F. Hudert, C. Janke, T. Dekorsy, and K. Kohler, Appl. Phys. Lett. 88, 041117 (2006).

8. J. Davila-Rodriguez, M. Bagnell, C. Williams, and P. J. Delfyett, J. Lightwave Technol. 29, 3091 (2011).

9. L. Antonucci, X. Solinas, A. Bonvalet, and M. Joffre, Opt. Express 20, 17928 (2012).

10. A. Fernandez, T. Fuji, A. Poppe, A. Fürbach, F. Krausz, and A. Apolonski, Opt. Lett. 29, 1366 (2004).

11. Since the time resolution of the experiment-limited by other factors discussed in the text-is more than five times longer than the specified laser pulse durations, an accurate measurement of the actual pulse durations at the time of the experiment was considered unnecessary.

12. M. H. Vos, J. Breton, and J.-L. Martin, J. Phys. Chem. 101, 9820 (1997).

13. W. W. Parson and T. G. Monger, Brookhaven Symp. Biol. 28, 195 (1977).

14. K. E. McAuley-Hecht, P. K. Fyfe, J. P. Ridge, S. M. Prince, C. N. Hunter, N. W. Isaacs, R. J. Cogdell, and M. R. Jones, Biochemistry 37, 4740 (1998).

15. K. Gibasiewicz, M. Pajzderska, M. Ziolek, J. Karolczak, and A. Dobek, J. Phys. Chem. B 113, 11023 (2009).

16. K. Gibasiewicz, M. Pajzderska, A. Dobek, K. Brettel, and M. R. Jones, "Analysis of the kinetics of P+HA- recombination in membrane-embedded wild-type and mutant Rhodobacter sphaeroides reaction centers between 298 and $77 \mathrm{~K}$ indicates that the adjacent negatively charged QA ubiquinone modulates the free energy of $\mathrm{P}+\mathrm{HA}-$ and may influence the rate of the protein dielectric response," J. Phys. Chem. B (to be published).

17. J.-L. Martin, J. Breton, A. J. Hoff, A. Migus, and A. Antonetti, Proc. Natl. Acad. Sci. USA 83, 957 (1986). 